\title{
Mejora de la cuantificación y armonización cuantitativa en PET mediante filtrado adaptativo
} \section{Improved PET quantification and harmonization by adaptive denosing}

Presentación: 6-7/10/2020

\section{Doctorando:}

\section{Mauro Namías}

Departamento de Física Médica, Fundación Centro Diagnóstico Nuclear, Buenos Aires, Argentina mauro.namias@gmail.com

\section{Director:}

\section{Robert Jeraj}

\section{Resumen}

La cuantificación en la tomografía por emisión de positrones (PET) está sujeta a sesgos debido a limitaciones físicas y técnicas. El objetivo de la armonización cuantitativa es lograr mediciones comparables entre diferentes tomógrafos PET, permitiendo así ensayos clínicos multicéntricos. Las guías clínicas, como las de la Asociación Europea de Medicina Nuclear (EANM), recomiendan armonizar las reconstrucciones de PET para llevar los coeficientes de recuperación de contraste (CRC) dentro de las especificaciones. Sin embargo, estas reconstrucciones armonizadas pueden mostrar sesgos cuantitativos. En este trabajo mejoramos la armonización mediante el uso de un novedoso esquema de filtrado adaptativo. Nuestro objetivo fue obtener un sesgo de cuantificación bajo y valores de relación señal / ruido (PSNR) altos al mismo tiempo.

Para ello se diseñó e implementó un nuevo filtro de eliminación de ruido adaptativo de tres etapas. Los parámetros del filtro se optimizaron para lograr un PSNR alto en un fantoma cerebral digital y un sesgo cuantitativo bajo de los valores máximos de recuperación de contraste (CRCmax) obtenidos de un fantoma de calidad de imagen de PET de la Asociación Nacional de Fabricantes Eléctricos (NEMA). El fantoma NEMA se escaneó en varios escáneres PET / CT y se reconstruyó sin filtros. La configuración de filtro óptima encontrada para un conjunto de datos de entrenamiento se aplicó luego a las reconstrucciones de prueba de otros escáneres. Los límites de armonización se definieron utilizando los intervalos de confianza del $95 \%$ entre reconstrucciones.

Como resultado, se lograron valores de CRCmax promedio cercanos a la unidad $( \pm 5 \%)$ para esferas con un diámetro igual o superior a $13 \mathrm{~mm}$ para el conjunto de datos de entrenamiento. Los valores de PSNR fueron comparables a otros resultados de filtros de última generación. Usando la misma configuración de filtro óptima para los conjuntos de datos de prueba, se encontraron resultados cuantitativos similares. La visibilidad de la lesión mejoró en imágenes clínicas en comparación con las reconstrucciones EANM, sin artefactos visibles. De esta forma, nuestro filtro adaptativo de tres etapas logró un rendimiento cuantitativo de vanguardia para la obtención de imágenes PET. Se lograron tolerancias de armonización con un sesgo y varianza más bajos que las directrices de EANM para una variedad de modelos de escáner. Los valores de CRCmax estaban cerca de la unidad y la variabilidad de cuantificación se redujo en comparación con las reconstrucciones estándar.

Palabras clave: PET, filtrado adaptativo, cuantificación, armonización

\section{Abstract}


Quantification in positron emission tomography (PET) is subject to bias due to physical and technical limitations. The goal ofquantitative harmonization is to achieve comparable measurements between different scanners, thus enabling multicenter clinical trials. Clinical guidelines, such as those from the European Association ofNuclear Medicine (EANM), recommend harmonizing PET reconstructions to bring contrast recovery coefficients (CRCs) within specifications. However, these harmonized reconstructions can show quantitative biases. In this work we improve harmonization by using a novel adaptive filtering scheme. Our goal was to obtain low quantification bias and high peak signal to noise ratio (PSNR) values at the same time.

For this purpose, a novel three-stage adaptive denoising filter was implemented. Filter parameters were optimized to achieve both high PSNRin a digital brain phantom and low quantitative bias ofmaximumCRC values (CRCmax) obtained from a National Electrical Manufacturers Association (NEMA) PET image qualityphantom. TheNEMAphantom was scanned on several PET/CT scanners and reconstructed without postfilters. The optimal filter settings found for a training dataset were then applied to testing reconstructions from other scanners. Harmonization limits were defined using the $95 \%$ confidence intervals across reconstructions.

Average CRCmax values close to unity $( \pm 5 \%)$ were achieved for spheres with diameter equal or greater than $13 \mathrm{mmfor}$ the training dataset. PSNR values were comparable to other state-of-the-art filter results. Using the same optimal filter settings for the testing datasets, similar quantitative results were found. Lesion conspicuitywas improved on clinical scans when compared withEANM reconstructions, with no visible artifacts. In this way, our three-stage adaptive filter achieved state-of-the-art quantitative performance for PET imaging. Harmonization tolerances with lower bias and variance tan EANM guidelines were achieved for a variety ofscanner models. CRCmax values were close to unityand the quantification variability was reduced when compared with standard reconstructions.

Keywords: PET adaptive denoising quantification harmonization

\section{Introducción}

La cuantificación de estructuras pequeñas generalmente no es comparable entre los diferentes modelos de escáner PET. Esto se debe a los diferentes diseños de detectores, algoritmos de reconstrucción, configuraciones de reconstrucción y filtros de suavizado, que afectan el contraste final y el ruido en las imágenes reconstruidas (Boellaard, 2009). Los filtros de suavizado se utilizan para reducir los niveles de ruido en las imágenes reconstruidas, a expensas de una reducción de la resolución espacial de un sesgo negativo en la cuantificación de estructuras pequeñas. El objetivo de la armonización cuantitativa en la PET es hacer que las mediciones sean comparables entre los diferentes modelos de escáner, lo que permite ensayos clínicos multicéntricos y el seguimiento del paciente en el entorno clínico. La asociación europea de medicina nuclear (EANM) propuso protocolos de armonización para lograr resultados cuantitativos comparables para diferentes sistemas de PET. Estos protocolos prescriben tolerancias para los coeficientes de recuperación de contraste (CRC), medidos a partir de fantomas de calidad de imagen corporal NEMA estándar. El CRC es el cociente entre la actividad medida en la imagen y la actividad real presente en la esfera, y este valor depende del tamaño de la estructura. Cuando el CRC es igual a uno, significa que la actividad medida es igual a la actividad real (es decir, sin sesgo cuantitativo).

Como alternativa a los filtros lineales de suavizado, se han propuesto algoritmos de reducción de ruido no-lineales que preservan la resolución de las imágenes de medicina nuclear, tales como los filtros de difusión anisotrópicos (Demirkaya, 2004), los filtros bilaterales (Hofheinz et al., 2011), los filtros de media no-local (NLM) (Dutta, Leahy, \& Li, 2013; Qi et al., 2016), filtros colaborativos de agrupado de bloques en el dominio wavelet (Kim, Ahn, Nam, \& Ra, 2015; Maggioni, Katkovnik, Egiazarian, \& Foi, 2013), filtros wavelet y curvelet (Bal, Banerjee, Sharma, \& Maitra, 2019; Le Pogam, Hanzouli, Hatt, Cheze Le Rest, \& Visvikis, 2013) y redes neuronales profundas (Gong, Guan, Liu, \& Qi, 2019). Estos filtros adaptativos se diseñaron para preservar los bordes y los detalles al tiempo que reducen el ruido y aumentan la relación de contraste a ruido para estructuras pequeñas en comparación con los filtros lineales pasabajos (por ejemplo: filtros gaussianos). 
En este trabajo, implementamos un nuevo filtro adaptativo de tres etapas especialmente diseñado para imágenes PET y estudiamos su impacto en las tolerancias de armonización cuantitativa para una variedad de escáneres PET. Además, evaluamos los sesgos de los valores CRCmax y el rendimiento de reducción de ruido para propósitos generales (PSNR).

\section{Desarrollo}

Se diseñó un filtro de reducción de ruido adaptativo de tres etapas, donde las salidas de cada etapa se utilizan para guiar la reducción de ruido de la siguiente etapa. De esta manera, cada etapa refina el resultado de la anterior. Aunque dos etapas de eliminación de ruido suelen ser la opción estándar para tareas de reducción de ruido de propósito general (Maggioni et al., 2013; Manjón, Louis Collins, Coupé, Robles, \& Buades, 2011), agregamos un filtro rotacionalmente invariante como tercera etapa para mejorar la cuantificación de estructuras pequeñas.

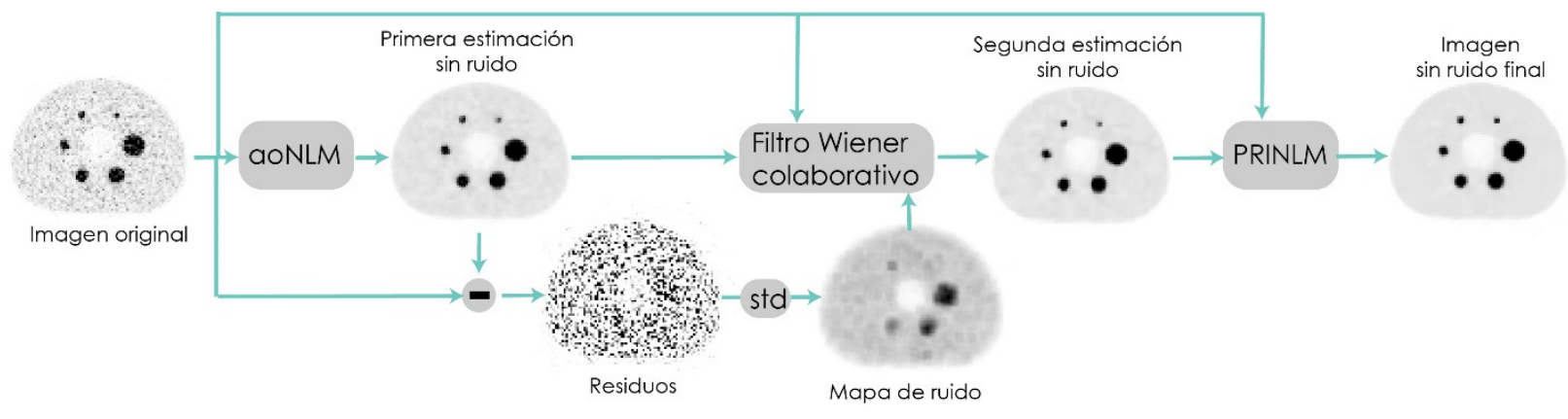

Figura 1. Diagrama en bloques del filtro adaptativo de tres etapas.

La primera etapa realiza una estimación inicial del nivel de ruido para cada vóxel y utiliza esta información para guiar un filtro de medias no locales (NLM) adaptativo y optimizado (AONLM) para reducir el ruido. La estimación del nivel de ruido se refina luego de la primera etapa restando la salida de esta etapa (primera estimación sin ruido) de la imagen original y luego estimando la desviación estándar de los residuos.

En la segunda etapa, se aplica un filtro de Wiener colaborativo a la imagen original, utilizando la salida de la primera etapa para guiar un proceso de block-matching. El filtro Wiener realiza una ponderación casi óptima de los coeficientes espectrales wavelet en función de la relación señal / ruido local, minimizando el error cuadrado medio (MSE) entre la imagen filtrada y la imagen original sin ruido (Levin \& Nadler, 2011; Milanfar, 2012). Utiliza la salida de la primera etapa como una estimación de la señal sin ruido y la desviación estándar local del ruido para calcular los pesos de Wiener en el dominio wavelet.

La última etapa consiste en un filtro NLM rotacionalmente invariante (PRINLM). Esta etapa se introdujo para mitigar las limitaciones de la invariancia rotacional de las etapas anteriores. En lugar de utilizar una métrica de distancia en bloques, implementa una métrica rotacionalmente invariante. Esto da como resultado un mayor rendimiento de eliminación de ruido para estructuras rotacionalmente invariantes, como esferas y bordes curvos.

Seleccionamos un fantoma digital del cerebro (Belzunce, 2018) para evaluar el rendimiento del PSNR, ya que el cerebro es una de las estructuras más complejas (ricas en información) que se puede estudiar con PET. Se agregó ruido correlacionado realista dependiente de la señal para simular un escaneo corto del cerebro de 1 minuto, usando la resolución y las mediciones del espectro de potencia de ruido (NPS) del escáner PET/CT GE Discovery 710 y la configuración de reconstrucción \# 1 de la tabla 1 (Namias, Bradshaw, Menezes, Machado, \& Jeraj, 2018). Las imágenes transaxiales de muestra del fantoma se muestran en la Figura 2.

Para evaluar el rendimiento de reducción de ruido en términos del sesgo de cuantificación de los valores CRCmax, escaneamos fantomas de calidad de imagen NEMA con insertos esféricos en múltiples tomógrafos PET. Los fantomas se llenaron con una solución de 18F-FDG, calibrada al inicio de la adquisición de la imagen, de $20 \mathrm{kBq} / \mathrm{ml}$ para las seis esferas y $2 \mathrm{kBq} / \mathrm{ml}$ para el fondo. Se obtuvieron imágenes del fantoma en los siguientes tomógrafos PET/CT: GE Discovery 710, GE Discovery IQ, GE Optima 560, GE Discovery STE y Siemens Biograph Truepoint TrueV. 

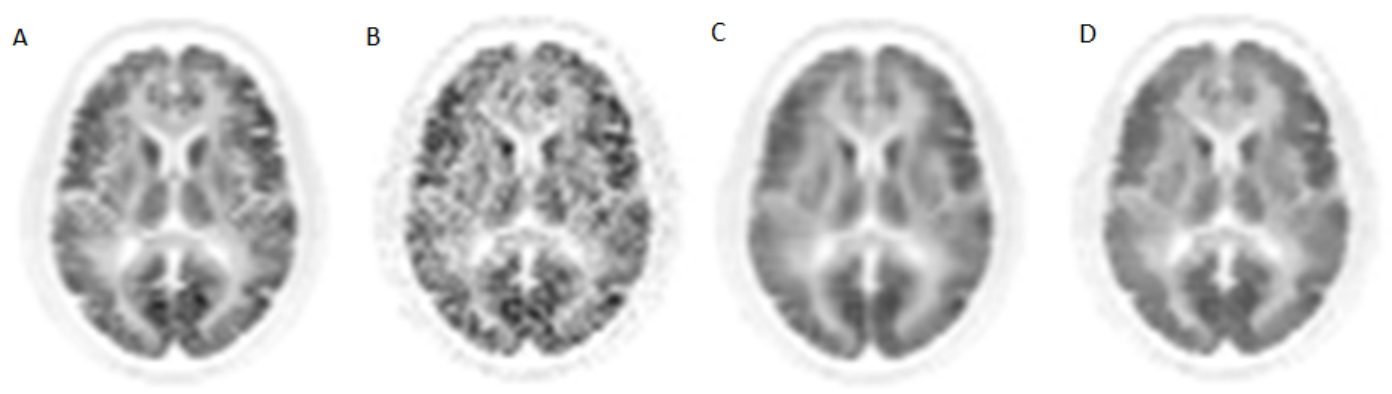

Figura 2. Imágenes transaxiales del fantoma cerebral. A) Imagen original sin ruido. B) Imagen con ruido, simulando una adquisición de 60 segundos (PSNR $=21.84$ dB). C) Resultados del filtro BM4D (PSNR = 24.94 dB). D) Resultado del filtro de tres etapas con parámetros óptimos (PSNR $=24.91 \mathrm{~dB})$.

En este trabajo, nos enfocamos en optimizar los macroparámetros de regularización de los filtros de media no local. Implementamos una estrategia simple de búsqueda en el espacio de los parámetros $\beta$ AONLM y $\beta$ PRINLM. Para cada par de valores, estimamos el valor de PSNR entre la imagen sin ruido del fantoma del cerebro, utilizando el fantoma sin ruido como referencia. Además, estimamos el error cuadrático medio (RMS) $\varepsilon$ de los valores CRCmax de las 5 esferas más grandes del fantoma NEMA para la misma configuración del filtro. También utilizamos el filtro BM4D (Maggioni \& Foi, 2011) como referencia para procesar ambos fantomas (cerebral y NEMA).

Después de procesar el fantoma cerebral con el filtro BM4D, se obtuvo un valor de PSNR de 24.94 dB (Figura 2). Para el filtro de tres etapas, se encontró un rendimiento de PSNR coincidente con el filtro BM4D para $\beta$ AONLM $=1$ y $\beta$ PRINLM $=0.30$.

Los valores de CRC después de aplicar la misma configuración de filtro óptima a todas las adquisiciones individuales de todas las configuraciones de reconstrucción se muestran en la Figura 3.
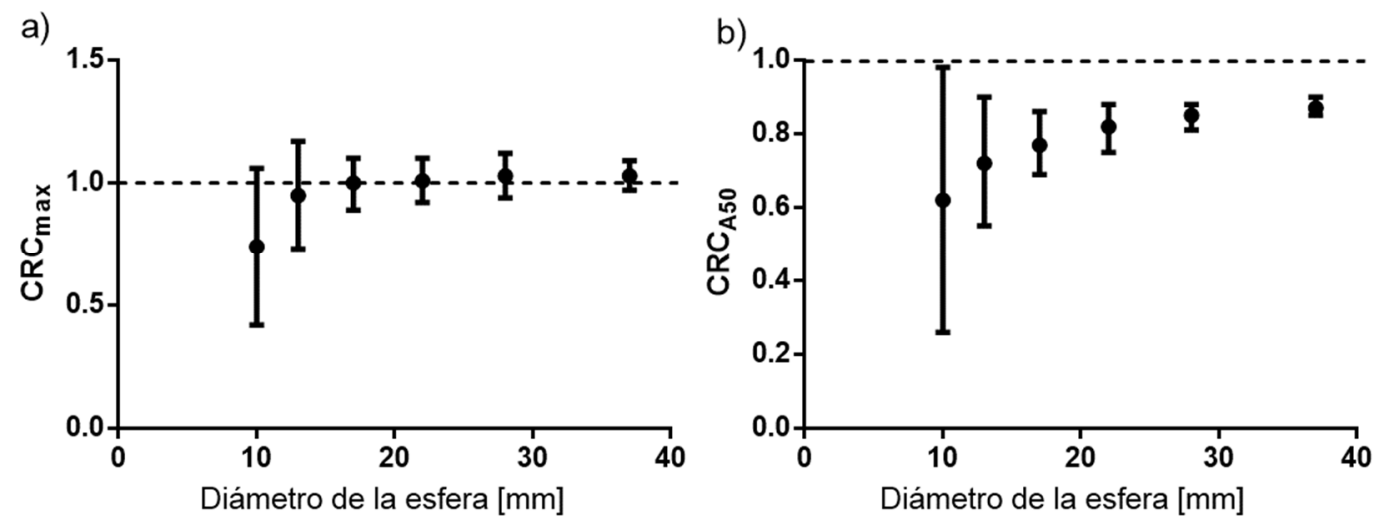

Figura 3. Armonización cuantitativa. a) Valores medios de $\mathrm{CRC}_{\max }$ (círculos) e intervalos de confianza del $95 \%$ (barras de error, IC) para todas las adquisiciones individuales (40 adquisiciones) de todos los tomógrafos. b) Valores medios de CRCA50 (círculos) e IC del 95\%.

En la Figura 4 se muestra una comparación gráfica de estas tolerancias.

Los resultados para la imagen clínica procesada con la configuración óptima del filtro y la configuración armonizada de EANM se muestran en la Figura 5. Obsérvese el aumento de la visibilidad de la lesión y el nivel de ruido reducido del filtro adaptativo en comparación con las reconstrucciones de EANM. No se observaron artefactos visibles para los resultados del filtro adaptativo. 

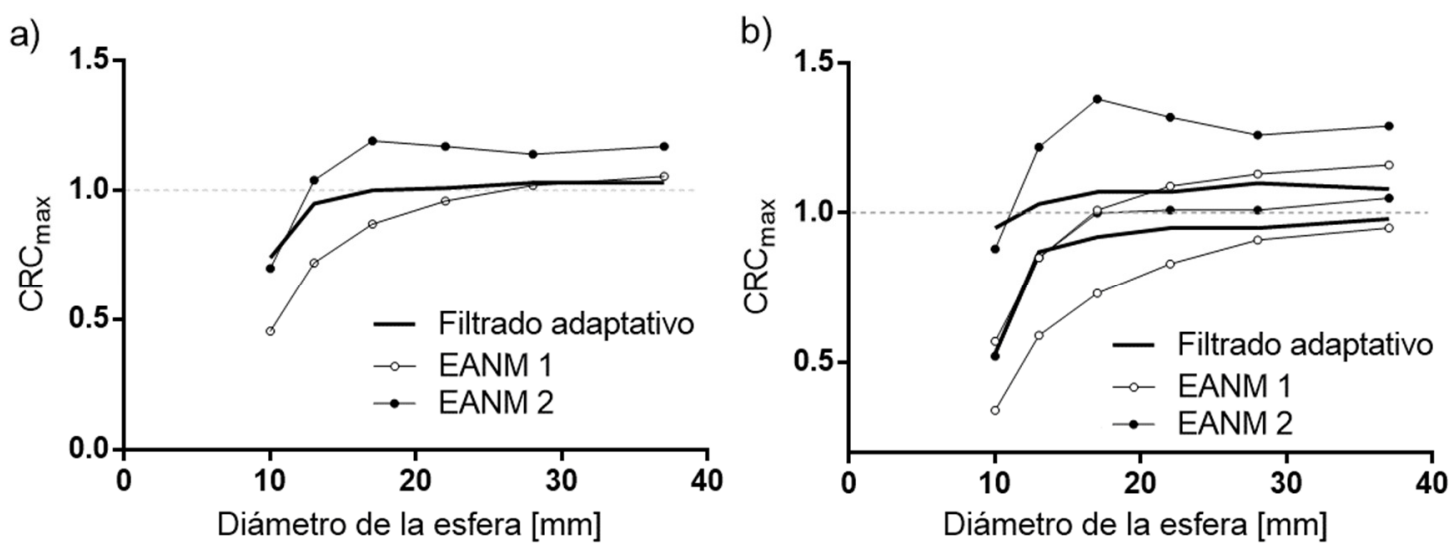

Figura 4. Comparación de diferentes tolerancias de cuantificación para (RCmax. a) Los valores promedio de las tolerancias de armonización se estimaron como el promedio de las tolerancias mínimas y máximas de (RCmax para cada tamaño de esfera. b) Tolerancias (límites mínimos y máximos). Los valores promedio y de ancho de banda se dividieron en dos gráficos diferentes para mejorar su legibilidad.

a)
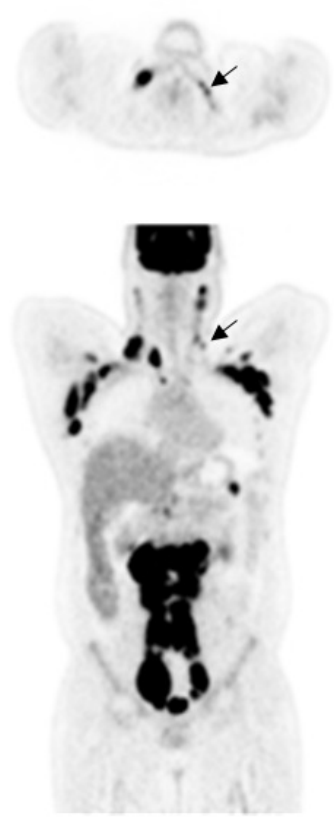

b)
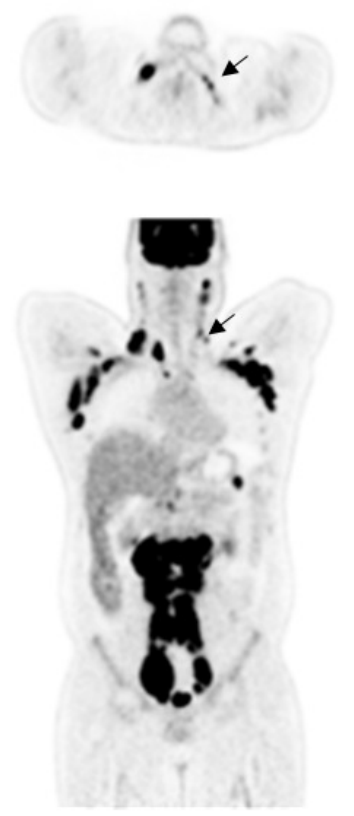

c)
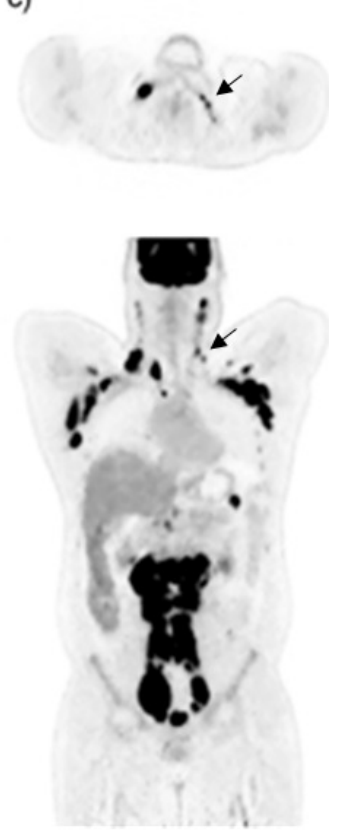

Figura 5. Cortes axiales y coronales de una imagen clínica de 18F-FDG. Armonizado con las especificaciones (a) EANM-1 y (b) EANM-2. (c) Filtrado con el filtro adaptativo de 3 etapas con parámetros óptimos. La visibilidad de la lesión (flecha) del filtro adaptativo (FA) fue mayor que para las reconstrucciones EANM (SUVmax = 10.6 g/ml, $12.9 \mathrm{~g} / \mathrm{ml}$ y $14.0 \mathrm{~g} / \mathrm{ml}$ para EANM-1, EANM-2 y FA respectivamente), mientras que el hígado el ruido fue menor ( $C V=8.0 \%, 8.4 \%$ y $5.1 \%$ para EANM-1, EANM-2 y AD respectivamente).

Los resultados cualitativos muestran que la visibilidad de las lesiones pequeñas mejoró para el filtro adaptativo en comparación con las reconstrucciones armonizadas con EANM, incluso en comparación con la reconstrucción de EANM-2 que se basa en el modelado de PSF. El ruido hepático fue menor para el filtro adaptativo en comparación con las reconstrucciones EANM. Dado el aumento de la visibilidad de la lesión con una disminución simultánea del ruido de fondo, es probable que mejore la relación de compromiso entre el sesgo de cuantificación y la reproducibilidad y la detectabilidad de lesiones (Boellaard, 2013) con la estrategia de reducción de ruido propuesta. Esta hipótesis debe explorarse en futuros estudios de detección de lesiones.

El uso del filtro adaptativo de tres etapas propuesto para armonizar las reconstrucciones de diferentes modelos de escáner y configuraciones de reconstrucción fue factible, lo que resultó en menores sesgos y variabilidad de cuantificación en comparación con las especificaciones de armonización propuestas previamente (Kaalep et al., 2018). Los resultados más exactos y 
reproducibles logrados con la metodología propuesta deberían mejorar la cuantificación del SUV, mejorar la evaluación de la respuesta al tratamiento, la diferenciación de las lesiones benignas y malignas y la determinación del pronóstico del paciente.

\section{Conclusiones}

En este trabajo diseñamos, implementamos y validamos un filtro adaptativo para reducción de ruido de imágenes PET. El filtro adaptativo de tres etapas propuesto logró un rendimiento cuantitativo de estado del arte en términos de relación señal-ruido pico (PSNR) y sesgo cuantitativo. Se lograron tolerancias de armonización con sesgos y varianzas menores que los protocolos anteriores para una variedad de modelos de escáner. Los valores de $\mathrm{CRC}_{\max }$ estuvieron cerca de la unidad y la variabilidad de cuantificación también se redujo en comparación con las reconstrucciones estándar.

\section{Referencias}

Bal, A., Banerjee, M., Sharma, P., \& Maitra, M. (2019). An efficient wavelet and curvelet-based PET image denoising technique. Medical \& Biological Engineering \& Computing, 57(12), 2567-2598. https://doi.org/10.1007/s11517-019-02014-w

Belzunce, M., \& Reader, A. J. (2018). High-Resolution Heterogeneous Digital PET Brain Phantom based on the BigBrain Atlas. In 7 th Conference on PET/MR and SPECT/MR.

Boellaard, R. (2009). Standards for PET image acquisition and quantitative data analysis. Journal of Nuclear Medicine : Official Publication, Society of Nuclear Medicine, 50 Suppl 1(Suppl 1), 11S-20S. https://doi.org/10.2967/jnumed.108.057182

Boellaard, R. (2013). Optimisation and harmonisation: Two sides of the same coin? European Journal of Nuclear Medicine and Molecular Imaging, 40(7), 982-984. https://doi.org/10.1007/s00259-013-2440-9

Demirkaya, 0. (2004). Post-reconstruction filtering of positron emission tomography whole-body emission images and attenuation maps using nonlinear diffusion filtering. Academic Radiology, 11(10), 1105-1114. https://doi.org/10.1016/j.acra.2004.07.012

Dutta, J., Leahy, R. M., \& Li, Q. (2013). Non-local means denoising of dynamic PET images. PLoS ONE, 8(12). https://doi.org/10.1371/journal.pone.0081390

Gong, K., Guan, J., Liu, C.-C., \& Qi, J. (2019). PET Image Denoising Using a Deep Neural Network Through Fine Tuning. IEEE Transactions on Radiation and Plasma Medical Sciences, 3(2), 153-161. https://doi.org/10.1109/TRPMS.2018.2877644

Hofheinz, F., Langner, J., Beuthien-Baumann, B., Oehme, L., Steinbach, J., Kotzerke, J., \& van den Hoff, J. (2011). Suitability of bilateral filtering for edge-preserving noise reduction in PET. EJNMMI Research, 1(1), 1-9. https://doi.org/10.1186/2191219X-1-23

Kaalep, A., Sera, T., Rijnsdorp, S., Yaqub, M., Talsma, A., Lodge, M. A., \& Boellaard, R. (2018). Feasibility of state of the art PET/CT systems performance harmonisation. European Journal of Nuclear Medicine and Molecular Imaging, 45(8), 1344-1361. https://doi.org/10.1007/s00259-018-3977-4

Kim, J. H., Ahn, I. J., Nam, W. H., \& Ra, J. B. (2015). An Effective Post-Filtering Framework for 3-D PET Image Denoising Based on Noise and Sensitivity Characteristics. IEEE Transactions on Nuclear Science. https://doi.org/10.1109/TNS.2014.2360176

Le Pogam, a., Hanzouli, H., Hatt, M., Cheze Le Rest, C., \& Visvikis, D. (2013). Denoising of PET images by combining wavelets and curvelets for improved preservation of resolution and quantitation. Medical Image Analysis, 17(8), 877-891. https://doi.org/10.1016/j.media.2013.05.005

Levin, A., \& Nadler, B. (2011). Natural image denoising: Optimality and inherent bounds. In CVPR 2011 (pp. 2833-2840). IEEE. https://doi.org/10.1109/CVPR.2011.5995309

Maggioni, M., \& Foi, A. (2011). Nonlocal transform-domain denoising of volumetric data with groupwise adaptive variance estimation. IEEE Transactions on Medical Imaging, 22(1), 119-133. https://doi.org/10.1117/12.912109

Maggioni, M., Katkovnik, V., Egiazarian, K., \& Foi, A. (2013). Nonlocal transform-domain filter for volumetric data denoising and reconstruction. IEEE Transactions on Image Processing, 22(1), 119-133.

Manjón, J. V., Louis Collins, D., Coupé, P., Robles, M., \& Buades, A. (2011). New methods for MRI denoising based on sparseness and self-similarity. Medical Image Analysis, 16(1), 18-27.

Milanfar, P. (2012). A Tour of Modern Image Filtering: New Insights and Methods, Both Practical and Theoretical. IEEE Signal Processing Magazine, 30(1), 106-128. https://doi.org/10.1109/msp.2011.2179329

Namias, M., Bradshaw, T., Menezes, V. O., Machado, M. A. D., \& Jeraj, R. (2018). A novel approach for quantitative harmonization in PET. Physics in Medicine and Biology, 63(9), 569-569. https://doi.org/10.1088/1361-6560/aabb5f

Qi, W., Xia, T., Niu, X., Ji, C., Winkler, M., Asma, E., \& Wang, W. (2016). A non-local means post-filter with spatially adaptive filtering strength for whole-body PET. 2015 IEEE Nuclear Science Symposium and Medical Imaging Conference, NSS/MIC 2015, (1), 0-2. https://doi.org/10.1109/NSSMIC.2015.7582060 